\title{
Testing inter-relations between disturbed sleep and sterility in intra-specific hybrids of fruit fly
}

\author{
Lyudmila Zakharenko \\ Department of Insect Genetics \\ Institute of Cytology and Genetics \\ SB RAS, Novosibirsk, Russia \\ 0000-0002-6341-8522
}

\author{
Dmitriy Petrovskii \\ Department of Insect Genetics \\ Institute of Cytology and Genetics \\ SB RAS, Novosibirsk, Russia \\ 0000-0002-0623-0363
}

\author{
Arcady Putilov \\ Research Institute for Molecular Biology \\ and Biophysics \\ Federal Research Centre for Fundamental \\ and Translational Medicine \\ Novosibirsk, Russia \\ 0000-0003-2779-9046
}

\begin{abstract}
Recent research connected sleeping problems and infertility in women. Sleep-wake behaviors of humans and Drosophila melanogaster demonstrate similarity in responsiveness to thermal stress. Moreover, studies of dysgenic crosses of fruit flies revealed possibility of inducing sterility in female intra-specific hybrids exclusively in one of two cross directions and only by exposing hybrids to thermal stress. Such facts motivated us to utilize this fruit flies' model of temperature-sensitive sterility for examining interrelationship between deleterious effects of thermal stress on fertility, survival, and circadian patterns of locomotor activity and sleep. In three identical pilot experiments, these patterns were documented in $\mathbf{2 8 5}$ hybrids kept for, at least, five days in constant darkness at $20^{\circ} \mathrm{C}$ and $29^{\circ} \mathrm{C}$. Additionally, the rhythms of 30 hybrid females of the $70^{\text {th }}$ generation were tested. Sleep-wake patterns in females with temperatureinduced sterility (progeny of Canton-S females crossed with Harwich males) significantly differed from the patterns in three other (fertile) crosses (males born from the same parents and offspring obtained by crossing in opposite direction). However, a lower rather than higher sensitivity of infertile females to thermal stress was revealed, and it persisted over generations. Unlike three other crosses, infertile females did not demonstrate such typical for flies and humans' responses to heat as night sleep disturbance and delay of the evening peak of activity. An elongated "siesta" was the only typical response common for this and opposing female cross. Data on survival of 2000 flies indicated that survival rate of female hybrids of the $1^{\text {st }}$ and $70^{\text {th }}$ generations was the highest compared to the rates of parent strains and hybrid males. Future research of fruit flies' models of reproductive health might be purposed on exploring sleep patterns not only in their relation to developmental sterility but also in their relation to acute deleterious effect of thermal stress on fertility.
\end{abstract}

Keywords - infertility, reproductive health, sleep disorders, circadian rhythm, thermal stress, fruit fly model

\section{Introduction}

Relationship of infertility with circadian and sleep disturbances appears to be rather complex. Consequently, it is not well established so far. Complicating the issue is a question of whether circadian and sleep disturbances are the results of infertility, causes of infertility, or whether the relationship between them is one of reciprocal nature [1]. This makes difficult the therapeutic targeting both female reproductive function and sleep disorders. To answering the question of plausibility of causal relationship, it appears to be essential to understand genetic and environmental causes of association between infertility and disruptions of the fundamental circadian and sleep regulation mechanisms.

Given the complexity and limitations of human studies, the fruit fly, Drosophila melanogaster, is an ideal model organism for dissecting relationships between genes, environment, sleep-wake behavior, and various diseases [2]. It might, in particular, provide a powerful and rapid platform to study the mechanisms underpinnings the associations between environmental stress, infertility, and sleep-wake disturbances. Hot ambient temperature belongs to the most powerful natural factors causing the profound disturbances of the human sleep-wake cycle. For instance, a much stronger annual rhythmicity of sleeping problems was reported by both newcomers and native residents of a region with hot summer temperature as compared to people living at higher latitudes who are seasonally exposed to extremely long and short photoperiod with mostly cold rather than hot air temperatures [3]. The experimental research indicates that fly's sleep-wake pattern might be reorganized by heat in a way that is very similar to human sleep-wake pattern response to an increase of ambient temperature, i.e., nighttime sleep and daytime activity usually decrease whereas daytime sleep and early night activity usually increase during exposure to thermal stress [4]. On the other hand, Drosophila melanogaster can additionally serve as a model of temperature-sensitive agametic sterility that was originally called gonadal dysgenesis sterility [5]. Dysgenesic phenomena can occur exclusively in one of two cross directions and only due to cultivation under elevated temperature (reviewed in [6]). Therefore, the effects of thermal stress on the circadian rhythms in sterile female hybrids might be compared with the effects on these rhythms in the reciprocal (fertile) female hybrids. Consequently, the major aim of our pilot study was to utilize this fruit fly model of temperature-sensitive infertility for delineating possible causal link between the deleterious effects on fertility and sleep-wake behaviors imposed by such environmental factor as hot temperature. The hypothesis was that the circadian patterns of locomotor activity and sleep might be more dramatically disturbed by thermal stress in infertile rather than fertile crosses.

\section{Methods}

\section{Hybrids and Conditions}

In order to create hybrid females showing intra-specific paternal-maternal hybrid gonadal dysgenesis, females of Canton-S strain were crossed with males of Harwich strain, and F1 hybrids were maintained at high air temperature (i.e., eggs were hatched, larvae were reared, and newly emerged flies were kept permanently at $29{ }^{\circ} \mathrm{C}$ ). The reciprocal (fertile) female hybrids and fertile hybrid males served as three control crosses cultivated in the same environmental condition. During three different seasons, all flies were cultivated at $29{ }^{\circ} \mathrm{C}$ until age of, at least, 5 days in the laboratory to perform three experimental studies of their 
circadian patterns of locomotor activity and sleep. Additionally, female hybrids of two cross directions were reproduced for 70 generations at room temperature. In the $4^{\text {th }}$ season they serve two more controls for the experimental study of circadian patterns of F1 female hybrids.

Sleep Recordings

To document circadian rhythmicity and sleep, flies were placed individually in glass locomotor-monitoring tubes. The measurements were obtained simultaneously for equal in size subsamples of flies kept in constant darkness at either $20^{\circ} \mathrm{C}$ or $29^{\circ} \mathrm{C}$ during, at least, 5 days. Locomotor activity was monitored in 1-min bins using the Drosophila Activity Monitoring System ("Trikinetics", Waltham, MA, USA). Sleep events were defined as 5 consecutive minutes of absence of any locomotor activity [7]. Parameters of locomotor activity and sleep were calculated using data acquisition software package (www.trikinetics.com). Number of beam breaks and duration of sleep episodes were summed on 30-min intervals of 5-day record to draw illustrations and to perform statistical analyses with the SPSS $_{23.0}$ statistical software package (IBM, Armonk, NY, USA). The first day data were excluded, whereas the following 30-min estimates of activity or sleep were averaged on 4 24-h intervals to obtain measurements at each of 48 time points constituting the 24-hour cycle (Fig. 1).

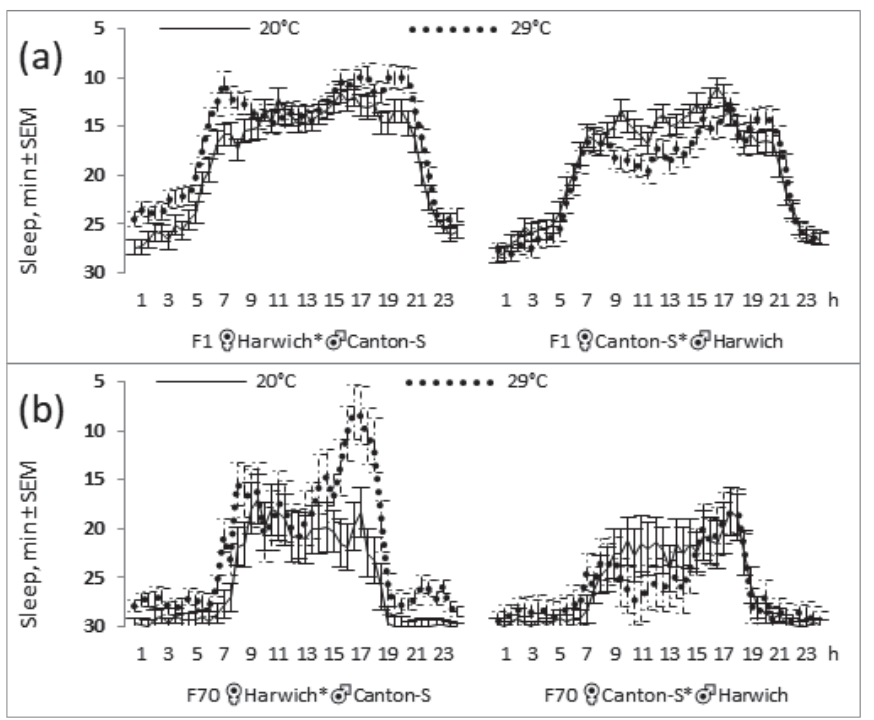

Fig. 1. 24-hour time courses of sleep in hybrid females of opposing cross directions (females from Harwich strain and males from Canton-S strain and females from Canton-S strain and males from Harwich strain). F1 and F70: crosses of either the $1^{\text {st }}$ (a) or $70^{\text {th }}$ generation (b) kept under either low $\left(20^{\circ}\right)$ or high air temperature $\left(29^{\circ}\right)$

\section{Survival Rate}

We also examined differences in survival rate under thermal stress in two parent strains and their F1 or F70 offspring of two cross directions. Flies were placed in tubes and kept at $29^{\circ} \mathrm{C}$. Food was replaced with intervals of, at least, 2 days and dead flies were removed and counted. In total, 2000 flies from 100 tubes were counted and mortality analysis was performed using the Kaplan-Meier estimator.

\section{Results}

As expected, sterility was found only in F1 females from one of two cross directions: when females from Canton-S strain were hybridized with males from Harwich strain. Moreover, the deleterious effect was found only when eggs were hatched, larvae were reared, and newly emerged flies were permanently kept at $29^{\circ} \mathrm{C}$. The main reason for sterility of this female cross was under-development of ovaries.

The crosses demonstrated significant difference in 24-h sleep-wake cycle, and in its response to thermal stress. For example, only fertile F1 females (i.e., progeny of Harwich females crossed with Canton-S males) responded to an elevated temperature by 1) a decrease of night sleep (an insomnia-like symptom), 2) a shift of the second activity peak from evening to early night hours, and 3) an increase of daytime sleep duration (it is typical for males under any temperature). In contrast, sterile F1 females demonstrated only one - the latter - response (see Figure 1,a). The same differences in sleep pattern and its stress response were also found in F70 females (see Figure 1,b). Longevity analyses yielded significant differences in survival rate indicating that female F1 or F70 offspring of any cross direction were significantly better survivals than females from any of two parent strains and any males.

\section{Discussion}

Given the complexity of human reproduction system and numerous limitations imposed on human studies, a fruit fly model was used to investigate the possible association between the predicted effects of thermal stress on the sleepwake cycle and fertility. Although no evidence was provided for a causal link between temperature-induced sterility and insomnia-like symptoms in females born from females of Canton-S strain and males of Harwich strain, the circadian sleep patterns and their responsiveness to thermal stress were found to be significantly dependent upon cross direction. This dependence was observed in intra-specific hybrids of any sex and persisted in females over generations. Future studies of fruit fly models of temperature-sensitive infertility might be focused on testing possibility of association between the acute rather than developmental thermal effects on fertility and sleep-wake patterns.

\section{ACKNOWLEDGMENT}

The fruit fly study of L. P. Z. and D. V. P. was partially funded by the Federal Research Program via project number 0324-2018-0016, and A. A. P. was partially funded by the Russian Foundation for Basic Research through a research grant number 19-013-00424.

\section{REFERENCES}

[1] N. D. White, "Influence of sleep on fertility in women," Am. J. Lifestyle Med., vol. 10, pp. 239-241, 2012.

[2] N. C. Donelson and S. Sanyal, S. "Use of Drosophila in the investigation of sleep disorders," Exp. Neurol., vol. 274 (Pt A), pp. 72-79, 2015

[3] A. A. Putilov, "A cross-sectional study of retrospectively reported seasonality in native and non-native residents of Chukotka and Turkmenistan," Int. J. Occup. Environ. Health, vol. 24, pp. 17-26, 2018.

[4] K. M. Parisky, J. L. Agosto Rivera, N. C. Donelson, S. Kotecha and L. C. Griffith, "Reorganization of sleep by temperature in Drosophila requires light, the homeostat, and the circadian clock", Curr. Biol., vol. 26, pp. 882-892, 2016.

[5] M. G. Kidwell, J. F. Kidwell and J. A. Sved, "Hybrid dysgenesis in Drosophila melanogaster: a syndrome of aberrant traits including mutation, sterility and male recombination," Genetics, vol. 86, pp. 813-833, 1977.

[6] W. R. Engels, "The P family of transposable elements in Drosophila," Ann. Rev. Genet., vol. 17, pp. 315-344, 1983. 